\title{
CONOCIMIENTOS Y SABERES EN DISPOSITIVOS PEDAGÓGICOS LA FORMACIÓN POLÍTICA EN MOVIMIENTOS POPULARES
}

\author{
María Mercedes Palumbo
}

\section{Resumen}

El objetivo de este artículo se centra en la recuperación y el análisis de los conocimientos y saberes convocados en dispositivos pedagógicos de formación política en movimientos populares. Allí se teje una compleja y heterogénea trama epistémica que involucra conocimientos académicodisciplinares, conocimientos académico-escolares y saberes populares. La metodología de estudio de casos múltiples contempló la triangulación de técnicas cualitativas en tres talleres de formación política desarrollados por movimientos populares del área metropolitana de Buenos Aires, Argentina. Los resultados obtenidos señalan la riqueza epistémica en circulación en estos dispositivos, que adoptan características singulares en función del modo en que los conocimientos $y$ saberes fueron puestos en juego.

Palabras clave: saberes, conocimientos, dispositivos pedagógicos, formación política, movimientos populares.

\section{Abstract}

Knowledge and wisdoms in pedagogical apparatus. Political formation in popular movements

This article is aimed at documenting and analyzing knowledge and wisdoms convoked in pedagogical apparatus of political formation in popular movements. A complex and heterogeneous epistemic frame emerges there that involves academic-disciplinary knowledge, academicscholastic knowledge and popular wisdoms. The multiple-case studies methodology considered the triangulation of qualitative methods within three political formation workshops developed in popular movements of Buenos Aires metropolitan area, Argentina. The results obtained show the epistemic diversity in such apparatus, assuming singular characteristics based on the way in which knowledge and wisdoms were compromised.

Keywords: wisdoms, knowledges, pedagogical apparatus, political formation, popular movements.

\footnotetext{
María Mercedes Palumbo: Doctora en el área Ciencias de la Educación, magíster en Educación, Pedagogías Críticas y Problemáticas Socioeducativas y licenciada y profesora en Ciencia Política por la Universidad de Buenos Aires (UBA). Investigadora asistente del Consejo Nacional de Investigaciones Científicas y Técnicas (CONICET). Docente del Seminario de Investigación sobre la Práctica Educativa de la Facultad de Ciencias Sociales de la UBA y del Seminario Planificación y Metodología de la Animación Social y Cultural del Departamento de Ciencias de la Educación de la Universidad Nacional de Luján. ORCID iD: 0000-0002-9765-1293

Email: mer.palumbo@gmail.com
}

Recibido: 15 de febrero de 2020

Aprobado: 21 de abril de 2020 


\section{Introducción}

La inquietud por la dimensión epistémica en las dinámicas de formación impulsadas desde los movimientos populares resulta clave frente al imperativo de recuperar y resignificar el valor del conocimiento como herramienta de lucha y su papel teórico-práctico en la construcción política. Por un lado, la disputa epistémica para los sujetos colectivos que no consienten con la hegemonía imperante se vuelve un tema sensible, con vistas a la generación y difusión de visiones del mundo alternativas a las de las usinas de producción y reproducción del pensamiento hegemónico. Adicionalmente, la cuestión epistémica interviene en la tramitación de las relaciones de saber-poder internas a los movimientos, donde también opera la significación simbólica de los conocimientos socialmente validados y se disponen fuentes epistémicas legítimas.

En ese marco general, el objetivo de este artículo se centra en la recuperación y el análisis de los conocimientos y saberes convocados en dispositivos pedagógicos de formación política de movimientos populares del área metropolitana de Buenos Aires (AMBA), Argentina. Puntualmente, se documentan las experiencias de los encuentros de formación del Movimiento Popular La Dignidad (MPLD), la Escuela de la Memoria Histórica (EMH) del Movimiento Nacional Campesino-Indígena de Buenos Aires (MNCI-Buenos Aires) y la "escuelita" de formación del Movimiento Darío Santillán (MDS), llevadas adelante en el período 2011-2015. En estos dispositivos se teje una compleja y heterogénea trama epistémica que involucra conocimientos académico-disciplinares, conocimientos académico-escolares y saberes populares. A modo de hipótesis interpretativa, asumimos que los modos de puesta en juego de los conocimientos y saberes disponen y jerarquizan ciertas fuentes epistémicas en el interior de los movimientos, otorgando singularidad a los dispositivos pedagógicos.

Los movimientos populares aquí estudiados se inscriben en la trayectoria abierta por los movimientos de trabajadores desocupados surgidos hacia finales de la década de 1990 en Argentina. Siguiendo a Maldovan Bonelli, Fernández Mouján e Ynoub (2019), el carácter territorial expresado en el sentido barrial y la vinculación con el trabajo - aun por la negativa, como trabajadores desocupados - fueron dos características que marcan el derrotero de estas organizaciones hasta la actualidad. A medida que fue avanzando la primera década del siglo XXI, nutridos por esta experiencia 
organizativa y en un marco de recomposición económica en el contexto de los denominados gobiernos kirchneristas, ampliaron sus demandas, repertorios de acción, sectores y sujetos involucrados, convirtiéndose en movimientos multisectoriales. En los últimos años, estos mismos movimientos entraron en un proceso de articulación en torno a la economía popular, desde el cual exigen el reconocimiento de la condición de trabajadores de los sectores no asalariados y disputan la redistribución de recursos, derechos y protecciones. A este respecto, la fundación de la Confederación de Trabajadores de la Economía Popular (CTEP), en el año 2011, marca un hito de la reconfiguración identitaria de estos sujetos colectivos en clave de organizaciones de la economía popular, combinando la originaria tradición movimentista con una vocación sindical. No obstante - y teniendo en cuenta el recorte temporal del desarrollo del trabajo de campo que dio lugar a este artículo-, las referencias a la economía popular aparecieron solo de un modo incipiente en los talleres de formación observados.

Cabe señalar que ya desde su génesis estos movimientos plantearon la formación política como tema de debate, práctica más o menos sistemática y principio de acción. La centralidad adjudicada a la formación se expresó igualmente en la existencia de un corpus académico abocado a la cuestión, que se fue consolidando al ritmo de la potencia pedagógica de sus referentes empíricos. Para el caso de Argentina, se destacan los estudios referidos a formas escolares gestionadas por los movimientos, como los bachilleratos populares de raigambre urbana (Ampudia y Elisalde, 2015; Aguiló y Wahren, 2014; Gluz, 2013) y la Escuela de Agroecología del Movimiento Campesino de Santiago del Estero (MOCASE-VC) (Burgos, 2013; Guelman, 2015; Michi, 2010a); producciones que abordan los procesos formativos en la propia cotidianeidad de la participación en un movimiento popular (Di Matteo, 2016; Di Matteo, De Mingo y Vila, 2012; Michi, 2010a, 2010b); y, finalmente, investigaciones abocadas a espacios intencionalmente formativos, aunque no escolares, que asumen generalmente la forma taller (Palumbo, 2017; Bruno, 2016; Korol, 2007; Rigal, 2015).

Considerando la vasta experiencia pedagógica de los movimientos populares, que combina instancias formales con otras informales de formación, aquí interesan específicamente los talleres de formación política. Su existencia responde al interés por socializar idearios, esquemas, vocabulario y proyectos estratégicos que abonen a la organicidad, coherencia e integralidad en las prácticas militantes. La adopción de la forma taller para llevar adelante esta formación da cuenta de una regularidad en la frecuencia de encuentros, así como de un grado considerable de planificación de las actividades en lo relativo a la delimitación de objetivos, contenidos, producción y reproducción de materiales de formación (cuadernillos, cartillas, textos y libros) y dinámicas de trabajo, aun siendo espacios de formación no escolares. Más allá de las particularidades asumidas por los tres talleres 
analizados, la metodología y el ideario de la educación popular aparecieron como rasgos comunes. A estos talleres asistieron, en carácter de destinatarios, militantes de base, mayoritariamente "compañeros de los barrios" (es decir, pertenecientes a las barriadas populares), junto a otros "compañeros nuevos", que eran estudiantes universitarios de clase media, en línea con la condición multisectorial que define a los movimientos en estudio.

La presentación de los contenidos se inicia con las coordenadas metodológicas de la investigación que sustenta las consideraciones de este artículo. A continuación, se recupera la perspectiva teórica que entrelaza las nociones de dispositivo pedagógico y ecología de saberes en el contexto epistémico propio de los movimientos populares multisectoriales. Luego, se aborda la configuración de los tres dispositivos de formación política en estudio, a partir de la revisita a los conocimientos y saberes convocados, dando lugar alternativamente a dispositivos textuales o narrativo-experienciales. Las reflexiones finales giran en torno a la riqueza epistémica en circulación en los dispositivos y los desafíos que de allí emergen.

\section{Aspectos metodológicos}

La investigación realizada se apoya en un diseño de tipo cualitativo (Vasilachis de Gialdino, 2007). La investigación cualitativa consideró la lógica metodológica más pertinente para comprender en profundidad las dinámicas epistémicas en dispositivos pedagógicos de formación política. De este modo, se buscó un análisis intensivo de los procesos sociohistóricos y político-pedagógicos intervinientes en las formaciones.

Como parte de la estrategia metodológica general, se desarrolló un estudio de casos múltiples (Yin, 1994). Concretamente, se tomaron tres dispositivos de formación política pertenecientes al MPLD, el MNCI-Buenos Aires y el MDS. La estrategia de casos múltiples aportó a la reflexión teórica y a la generación conceptual por medio de la replicación y la extensión de casos particulares (Martínez Carazo, 2006). El criterio de selección de los casos combinó la existencia de contactos previos con informantes claves y de condiciones organizacionales que facilitaran la accesibilidad tanto como el cuidado en la elección de casos centrales (Forni, 2010) con carácter ejemplificador en función de su capacidad para particularizar el problema en estudio. Se buscó así la significatividad de los movimientos populares en el amplio arco de movimientos populares del AMBA, así como la centralidad de sus trayectorias de formación política.

Para la construcción de la base empírica, se llevó adelante una triangulación de datos y técnicas cualitativas basadas en la observación participante de los talleres, las entrevistas en profundidad a sus participantes (destinatarios y coordinadores) y el análisis de materiales de formación (cartillas, cuadernillos, planificaciones). Se procuró así una comprensión 
más completa del objeto, a partir de la integración de diversas perspectivas, la búsqueda de consistencia de los datos, la contextualización de los procesos y la complementación de los aportes de cada técnica, fundamentales en un estudio de casos, donde emerge el problema de la validez y la confiabilidad de los datos (Yin, 1994).

La intención de este artículo no es realizar una descripción in extenso de los tres casos de estudio ni avanzar en una comparación entre estos, a modo de un inventario de similitudes o divergencias. Más que detallar, puntualizar y comparar, el gesto intelectual apunta a la elaboración de un análisis conjunto de los conocimientos y saberes convocados en los dispositivos pedagógicos de formación política, partiendo de la información empírica colectada y aportada por los tres casos.

\section{Acerca de los dispositivos pedagógicos y la ecología de saberes}

El abordaje propuesto para los talleres de formación política requiere aproximarse a ellos en su condición de dispositivos pedagógico-epistémicos. Siguiendo las consideraciones de Varcellino (2017), pensar un objeto en términos de dispositivo conlleva no tanto el trabajo con una mera categoría teórica como una estrategia de pensamiento y lectura de lo social. Esta estrategia posee dos aspectos: en primer lugar, nos sitúa en la "maraña" (Deleuze, 1990) de múltiples y heterogéneas prácticas que conforman el dispositivo, siempre relacionales e históricas; en segundo lugar, nos coloca en la inestable tensión entre novedad y repetición, entre lo instituyente y lo instituido.

Para Agamben (2011), en línea con la definición foucaultiana, el dispositivo alude a las relaciones existentes entre un conjunto de elementos de distinto tipo (leyes, normativas, organización de espacio y tiempo, discursos habilitados). Tres son sus notas centrales: a) la heterogeneidad de elementos discursivos y no discursivos que lo constituyen, b) su inscripción en una relación de poder dentro de la cual cumple una función estratégica en clave de capturar, controlar y orientar gestos, conductas, opiniones y discursos; y c) la imbricación entre saber y poder que lo caracteriza. Siguiendo al autor,

“[...] para otorgar una generalidad más grande a la clase de por sí vasta de los dispositivos de Foucault, llamo dispositivo a todo aquello que tiene, de una manera u otra, la capacidad de capturar, orientar, determinar, interceptar, modelar, controlar y asegurar los gestos, las conductas, las opiniones y los discursos de los seres vivos." (2011, p. 257).

Si para Foucault el dispositivo por antonomasia era la prisión, Agamben amplía su definición mencionando explícitamente a la escuela y aquí la extendemos también a espacios de formación no escolares. 
En clave de extrapolación de esta definición general, Grinberg (2008) propone la noción de dispositivo pedagógico. Con este término, la autora nombra un conjunto diverso de prácticas, saberes, procesos y estrategias que se articulan en los espacios-momentos de formación, en el encuentro entre por los menos dos sujetos, donde se crean subjetividades, se distribuyen determinados tipos de conocimientos y saberes y se despliegan relaciones de saber-poder. Siendo el dispositivo - ante todo - una máquina que produce subjetividades, se convierte en una máquina de gobierno que orienta las conductas, produce efectos subjetivos, genera operaciones de poder y produce saberes. Por lo tanto, el dispositivo alude a una red de relaciones consolidadas.

La puesta en diálogo de la noción foucaultiana de dispositivo - y sus citadas reelaboraciones - con la epistemología crítica de Boaventura de Sousa Santos permite adentrarse en las implicancias de las estrategias de visibilidad e invisibilidad que competen a lo epistémico. Es decir, la puesta en juego de ciertos conocimientos y saberes en un dispositivo da cuenta de un proceso de selección, privilegio y legitimación epistémica. Según De Sousa Santos, lo "no existente es producido activamente como no existente" (2006, p. 23). La monocultura del saber y del rigor que equipara el conocimiento con el producto de la ciencia occidental, con el conocimiento fundado en las disciplinas del saber, amenaza la diversidad epistémica mediante la reducción de la realidad a lo existente. Así, la pluralidad de saberes populares que convive con el conocimiento científico, aunque no se atiene a sus cánones, es confinada a un lugar de subalternidad, carencia e "ignorancia", angostando el campo de la realidad epistémica relevante.

En los talleres de formación política, la convivencia entre conocimiento académico-disciplinar, conocimiento académico-escolar y saberes populares no opera en un vacío epistémico. La condición multisectorial de los movimientos populares en análisis no es un dato menor en lo que a su correlato epistémico refiere. La multisectorialidad da cuenta de la coexistencia de trayectorias formativas, militantes, laborales, migratorias, de clase y género, que se traducen en una diversidad de conocimientos y saberes presentes en los talleres de formación política. Por lo tanto, es una dimensión que potencia y tensiona las experiencias de formación. Potencia en tanto la heterogeneidad de trayectorias de los militantes coloca una trama compleja y heterogénea de conocimientos y saberes que podría habilitar la conformación de una comunidad epistémica más amplia, en línea con el concepto de ecología de saberes (De Sousa Santos, 2006). Una comunidad epistémica más amplia implicaría el enriquecimiento y la contribución a un proyecto político donde todos los conocimientos y saberes se tornan indispensables y valiosos. Como lo expresan Falero, Casas, Brenes, Rieiro y Rocco (2011), la formación en los movimientos es un ámbito de creación y transmisión de conocimientos sustantivos para su reproducción y proyección. Adicionalmente, la multisectorialidad genera tensiones, dado 
que el conocimiento académico-disciplinar, encarnado en ciertos militantes participantes de la formación —-sean coordinadores o destinatarios-, plantea la posibilidad de reproducción de su jerarquía epistémica, incluso en experiencias pedagógicas que podrían ser consideradas a priori como novedosas.

\section{Los conocimientos y saberes convocados por los dispositivos de formación política}

Los conocimientos y saberes en circulación en los dispositivos estudiados habilitan su clasificación, siguiendo a Mejía Jiménez (2015), en: i) conocimientos fundados en las disciplinas del saber asociadas a la ciencia, aquí referidos como conocimientos académico-disciplinares y conocimientos académico-escolares; y ii) saberes populares propios de las prácticas sociales y las sabidurías particulares.

A los primeros, una militante los denominó "librescos", ${ }_{1}^{1}$ evidenciando su condición ligada a lo académico, lo escrito y lo intelectual. Por su parte, los saberes populares fueron mencionados por un militante como saberes "por experiencia vivida", 2 en situación de entrevista cuando refirió que sus aportes a la formación provenían de su historia personal, sus vivencias y sus prácticas, diferenciándolos explícitamente de aquellos conocimientos surgidos de los libros. En igual sentido apuntan otras categorías nativas recuperadas, tales como la "universidad de la calle", el "colegio de la calle" y la "necesidad como gran escuela". ${ }^{3}$ Así, estas categorías poseen un carácter esencialmente localista, situacional y con baja sistematicidad, son vehiculizadas fundamentalmente mediante la oralidad y portan un énfasis práctico. A este respecto, Ceceña sostiene que los saberes populares se construyen en el "rumiar cotidiano" en tiempos muy dilatados y los define como saberes materiales: "[...] un saber de tacto, de contacto, de los sabores ${ }^{4}$ y los saberes, un saber con (el saber de la dominación es un saber sobre). Hay un saber ins-crito y no necesariamente es-crito" (2008, p. 23).

El contrapunto entre estos tipos de conocimientos y saberes, según la bibliografía especializada y las concepciones de los militantes participantes de la formación, discurre entre la escritura y la oralidad, lo conceptual y lo vivencial, la sabiduría de los libros y la sabiduría plebeya; en fin, entre teoría y práctica. Empero, desde nuestra perspectiva, es dable relativizar la rigidez

1 Carla, militante del MCC invitada a la EMH. Entrevista realizada el 17 de julio de 2014.

2 Carlos, militante de base del MDS. Entrevista realizada el 19 de septiembre de 2015.

3 Estas categorías son planteadas, respectivamente, por Facundo, del MPLD (entrevista realizada el 24 de julio de 2013); Ramona, Mirta e Irene, del grupo productivo de dulces del MNCI-Buenos Aires (entrevista realizada el 06 de junio de 2013); y Elsa del MPLD (entrevista realizada el 16 de noviembre de 2012).

4 La relación entre saberes y sabores ya se encuentra en la etimología latina de sapere, que remite, simultáneamente, a tener inteligencia y buen gusto. 
de esta clasificación como una total polarización, rescatando el carácter dinámico, impuro e incompleto de los saberes. En esta línea, es posible suponer que los saberes populares contienen sedimentos de conocimientos académico-disciplinarios a los que se accedió, por ejemplo, mediante la escuela o los medios de comunicación. De la misma manera, los conocimientos académico-disciplinares - especialmente aquellos seleccionados en los dispositivos analizados que responden a una tradición crítica comprometida con la práctica - incluyen huellas de saberes populares.

De la coexistencia de conocimientos "librescos" y saberes "por experiencia vivida" no debe seguirse su despliegue equitativo dentro de los tres dispositivos de formación. Mientras en la EMH de la que participó el MNCI-Buenos Aires se privilegiaron los saberes "por experiencia vivida", en el caso del MPLD y el MDS los conocimientos "librescos" ocuparon un papel estructurador de los contenidos. Como fuera planteado en la introducción, sostenemos la hipótesis interpretativa de que los modos en que los distintos tipos de conocimientos y saberes fueron movilizados en los talleres de formación política generaban configuraciones singulares de los dispositivos pedagógicos, tal como se analizará a continuación.

\section{Conocimientos "librescos" y dispositivos textuales}

A una de las formas posibles de configuración del dispositivo la denominamos textual —en su remisión al texto, a lo escrito- al considerar la primacía de la transmisión de conocimientos académico-disciplinares a la hora de estructurar el taller de formación política y, en paralelo, el énfasis en la recuperación y deconstrucción de conocimientos académico-escolares. Esta primacía se observó en los contenidos seleccionados, la elaboración, el uso y la elección de materiales escritos de apoyo a la formación y las trayectorias educativas de las coordinaciones y los sujetos invitados a la formación.

A este tipo de configuración respondieron el taller del MPLD y del MDS, aunque en este último caso el carácter estructurador de lo textual se combinó con una fuerte transmisión intergeneracional de una memoria de saberes populares de la lucha por parte de la coordinadora, que se abordará más adelante. En ambas formaciones, los conocimientos académico-disciplinares circularon a partir de la socialización de conceptos introductorios a la tradición marxista ligados a la economía política, la sociología y la ciencia política. No es casual la selección de estos contenidos, en tanto responden a los fundamentos político-ideológicos de estos movimientos. Otro conocimiento académico-disciplinar común a los dispositivos en estudio abrevó en la historia como disciplina, que se erigió en un contenido destacado en los talleres y, adicionalmente, operó como perspectiva de análisis de otros contenidos que requerían una inscripción en un marco témporo-espacial. Ahora bien, se planteó un modo de contar la historia basado en una deconstrucción de 
los conocimientos académico-escolares por medio de la socialización de contrahistorias que recuperaran perspectivas desde abajo y desde el pueblo.

La centralidad de la historia en la formación política es conceptualizada por Caldart (2004) con referente empírico en el Movimiento de los Trabajadores Sin Tierra (MST), aunque con posibilidad de extenderse a las prácticas de otros movimientos populares, como la gestación de una "pedagogía de la historia". Siguiendo a la autora, los movimientos se educan cultivando la memoria subversiva del pueblo y comprendiendo y valorizando la historia, con vistas a reforzar la sensación de pertenecer a una tradición de rebelión y a una historia más amplia que los trasciende y de la cual deben aprender, en contraposición a la tentativa de educar dentro de una postura presentista y antihistórica. En este sentido, "estudiar historia es más que estudiar historia porque es necesario adentrarse en este estudio en el propio proceso de enraizamiento en ella" (2004, p. 377).

La transmisión de estos contenidos se apoyó, para el caso del MPLD, en una serie de diez cuadernillos elaborados por la coordinación como un material específico para la formación, que fueron entregados a los participantes para la dinámica de trabajo presencial y como material a profundizar en el estudio autónomo fuera de la formación. Aquí cabe señalar que la impronta textual del dispositivo se vio reforzada por la participación de invitados-especialistas para que aportaran una mirada general del contenido en cuestión, por la condición universitaria de las coordinadoras y por el fomento de la producción escrita de los participantes. En el taller del MDS, se apeló a una selección del libro de divulgación de teoría marxista ¿Qué es la sociedad?, de Marta Harnecker, y del libro La clase trabajadora nacional, de Guillermo Gutiérrez, que propone una lectura histórica en clave nacionalista revolucionaria.

En cuanto a los conocimientos académico-escolares, la escuela irrumpió en los dispositivos pedagógicos en su calidad de forma y contenido disponible (Palumbo, 2017), como un reservorio de formas y saberes previos para todos los militantes participantes, independientemente de sus trayectorias escolares. Cabe aclarar que estas trayectorias se caracterizaron por su heterogeneidad en los tres talleres: militantes que no habían concluido el nivel primario o secundario, otros que se encontraban retomando sus estudios en esos dos niveles, algunos que habiendo terminado el secundario no accedieron a estudios superiores, militantes universitarios graduados y estudiantes.

En efecto, los conocimientos académico-escolares adquirieron un estatuto paradójico en su recuperación y paralela impugnación parcial en los talleres de formación. Por una parte, se presentaron como punto de partida portado por los militantes, en tanto saberes previos que no podían ser negados e, inclusive, urgían ser utilizados por las coordinaciones como conocimientos de mediación entre los conocimientos académico-disciplinares que se buscaba socializar y los saberes populares. Esto es así dado que los 
conocimientos académico-escolares constituían un piso epistémico más cercano a los militantes de extracción no universitaria. En este sentido, se registró una reposición ex post de ciertos contenidos de la alfabetización escolar (y también académica), paralela a la alfabetización política, para el abordaje de los materiales escritos propuestos en la formación: recuperar un vocabulario más amplio — no militante- para comprender los textos, distinguir entre ideas primarias y secundarias, explicar el pasaje de números arábigos a romanos (y viceversa) al momento de leer los siglos, hacerse del hábito de la lectura y brindar pautas para la búsqueda y el análisis crítico de la información dispuesta en la web. Para la transmisión de conocimientos académico-disciplinares, la formación política precisaba contar con $-\mathrm{y}$ reponer- elementos de lectura y análisis provistos por el dispositivo pedagógico escolar.

De otra parte, se buscó problematizar y deconstruir los conocimientos académico-escolares en sus contribuciones a la producción y reproducción de la hegemonía epistémica imperante. Ciertos militantes tomaban prestados del dispositivo escolar roles, hábitos y representaciones sobre la enseñanza y el aprendizaje, disponibles como productos de sus trayectorias escolares, que trasladaban a los talleres de formación política. De igual forma, se valoraban (o no) los conocimientos y saberes en circulación en los talleres, así como las relaciones entre los sujetos portadores de ellos desde las experiencias transitadas en el dispositivo escolar. A modo de ejemplo, tal como relataba una militante, ${ }^{5}$ la concepción respecto a que formarse es "sentarse y escuchar al que sabe" en una actitud de recepción del conocimiento impartido por un supuesto sujeto del todo-saber — que en el caso de los talleres recalaba en la coordinación individual o colectiva, según el caso- reenvía principal, aunque no exclusivamente, a la experiencia y las trayectorias escolares.

Es posible señalar que la configuración textual del dispositivo está dada por los conocimientos seleccionados para estructurar la formación, pero no exime la presencia de otros tipos de saberes. Los "saberes por experiencia vivida" irrumpieron en las intervenciones espontáneas de los militantes y también fueron alentados, recuperados y puestos en valor por las coordinaciones. Se observaron esfuerzos de las coordinaciones con vistas a relacionar los conocimientos académico-disciplinares - algunos de ellos con un alto grado de complejidad - con la vida cotidiana de los destinatarios. La intención de que el aporte de las "ciencias" se combinara con la recuperación e intercambio de saberes populares de los militantes aparece claramente planteada en situación de entrevista con una coordinadora del taller del MPLD:

"El espíritu es ese, que tiene que ver con laburar desde la recuperación de los saberes y las formas de pensar y de vivir de los compañeros que estamos

5 Marianela, militante de clase media universitaria del MDS, destinataria de la formación docente. Entrevista realizada el 4 de septiembre de 2015. 
en los espacios de formación, aportar desde las ciencias en todo caso a ese conocimiento para que después eso tenga una vuelta en la práctica en términos transformativos". ${ }^{6}$

En torno a la historia, en su doble condición de contenido y perspectiva de abordaje, se observó más vívidamente este encuentro de conocimientos y saberes de distinta procedencia. Allí se cruzaron la historia como disciplina científica, el conocimiento histórico de corte académico-escolar y los saberes populares relativos a acontecimientos vividos o recepcionados mediante la transmisión oral. En el caso del MDS, la lectura del libro de Guillermo Gutiérrez se nutrió de la recuperación y transmisión de saberes populares de la lucha por parte de la coordinadora perteneciente a la generación militante de los setenta, planteando una continuidad histórica de las experiencias del campo popular con la generación política posterior al 2001, propia de los destinatarios de la formación.

\section{Saberes "por experiencia vivida" y dispositivos narrativo-experienciales}

En los dispositivos de formación observados, los saberes populares provinieron de, al menos, tres fuentes a las que los militantes se refirieron explícitamente en los encuentros formativos y en las entrevistas: i) las vivencias cotidianas familiares o locales transmitidas oralmente de generación en generación; ii) las experiencias de supervivencia en los barrios; y iii) las prácticas y los sentidos de la organización colectiva, la militancia y las luchas populares pasadas y presentes. Estos saberes son aquí sistematizados como saberes cotidianos, saberes de la supervivencia y saberes de la lucha, respectivamente. Como sostiene Korol, "los saberes populares abarcan desde distintas formas de alimentarse y curarse, de contar la historia y las historias, de relacionarse con la naturaleza, de comunicarse, de expresarse artísticamente hasta múltiples formas de lucha" (2008, p. 188). Cabe aclarar que se sigue una perspectiva alejada de la asignación de una naturaleza inherentemente emancipadora de los saberes populares; a contramano, se los concibe como la coexistencia contradictoria - pero convivencia al fin - de dominaciones y posibilidad de emancipaciones. ${ }^{7}$

Los saberes cotidianos, provenientes de la historia familiar o local, se relacionaban con festividades, consejos para curar enfermedades mediante el uso de plantas medicinales, recetas sobre comidas típicas, prácticas técnico-

6 Marina, coordinadora de los encuentros de formación del MPLD. Entrevista realizada el 17 de mayo de 2013.

7 Si bien no es objeto de este artículo, para profundizar en esta concepción compleja resulta relevante la perspectiva gramsciana que abona al escudriñamiento de las relaciones estructurantes de los saberes populares a partir de los conceptos de "sentido común" (entramado a la hegemonía y las dominaciones) y "buen sentido" (que puede sedimentar una concepción del mundo emancipatoria). 
productivas, cuentos y leyendas que incluían máximas de conducta. En los relatos de los entrevistados, estos saberes remiten a sus hogares con una fuerte impronta femenina en la transmisión (madres, tías, abuelas). En el caso de la EMH de la que participó el MNCI-Buenos Aires, quedó en evidencia el hecho de que gran parte de los saberes cotidianos no surgen del contexto geográfico de las barriadas del AMBA sino de provincias del interior del país y países limítrofes que conforman los lugares de origen de los militantes o sus familias. De lo anterior se desprende una memoria de saberes (Guelman y Palumbo, 2015) que es nombrada y reactualizada en los talleres de formación y las prácticas militantes en general.

En el trascurrir del trabajo de campo, se registraron —explicitando aquí solo algunos ejemplos entre los muchos que se sucedieron- explicaciones de militantes peruanas y bolivianas sobre la preparación de quinoa, chancacas, alfeñiques y guarapo, ${ }^{8}$ aportes de normas de conducta y leyendas de la cultura quechua, como el Ama quilla, ama shua, ama llulla (no mentir, no robar y no ser ocioso), ${ }^{9}$ y saberes sobre técnicas de producción campesina.

Por su parte, los saberes de la supervivencia conforman aprendizajes estratégicos relativos a un saber-hacer para paliar la necesidad y garantizar la subsistencia en un medio hostil signado por la confluencia de punteros políticos, transas, ${ }^{10}$ organizaciones y movimientos populares y la política social estatal; saberes que, a menudo, se aprenden haciendo y viviendo en las barriadas populares del AMBA. En los registros de observación y las entrevistas, se presentaron asociados a estrategias de sobrevivencia, como en las referencias a "saber levantar una pared", ${ }^{11}$ traccionado por la necesidad de acceder a una vivienda, o "aprender a costurar" 12 para ganarse el sustento. Tal como sentenció una militante en su intervención en un momento de plenario de la formación del MPLD: "La necesidad nos obliga a saber" ${ }^{13} \mathrm{Si}$ los saberes cotidianos usualmente formaban parte de los contextos de origen de los militantes, los saberes de la supervivencia se mostraban emplazados en los escenarios de llegada de las trayectorias migratorias, donde se asistía a

8 Registro de observación de la "escuelita" de formación del MDS (12 de junio de 2015).

9 Registro de observación de la "escuelita” de formación del MDS (14 de agosto de 2015).

10 Esta expresión se utiliza para referir a los sujetos que están a cargo de la intermediación en el negocio de la venta de drogas, asociados a lo que se denomina en Argentina "narcomenudeo". Con frecuencia, estos sujetos pertenecen al mismo barrio y despliegan contactos con los punteros políticos o las fuerzas de seguridad.

11 El "aprender a levantar paredes" aparece como una garantía de la construcción y el mejoramiento de las viviendas en las barriadas y, al mismo tiempo, cuestiona la centralización de la experticia en el ingeniero. Registro de observación del MPLD (7 de noviembre de 2012).

12 En el relato de Andrea, militante de base del MNCI-Buenos Aires, coser se comenta como un saber impuesto por la necesidad de sustento material. Entrevista realizada el 10 de octubre de 2015.

13 María, militante de base del MPLD. Reconstrucción de su intervención en los encuentros de formación del MPLD. Registro de observación (7 de noviembre de 2012). 
una resignificación de los saberes cotidianos portados en el nuevo contexto, así como se producían nuevos saberes.

Un tipo particular remite a los saberes de la supervivencia vinculados a las formas de pensar y practicar la política. Desde las interpretaciones de los militantes que se encontraban formándose, estos saberes de la supervivencia de tipo político respondían a un pasado en el cual no se encontraban organizados políticamente y reenviaban, incluso, al momento de ingreso por "necesidad" a los movimientos de pertenencia actual. En efecto, los saberes de la supervivencia se presentan anclados a un pasado relativamente reciente que es puesto en suspenso frente a otras lógicas de la política — de compromiso, de colectividad—, propuestas por los movimientos en los cuales militan. Algunos de estos saberes que circularon en los dispositivos de formación, enunciados desde una mirada crítica, apuntaban a cuestiones ligadas a quedarse callado y evitar discutir con los referentes de las organizaciones para pasar desapercibido, reproducir los discursos políticamente correctos y las formas de actuar esperables dentro de los movimientos y mostrarse falsamente interesados ante los dirigentes. ${ }^{14}$ No obstante la denuncia de la persistencia de saberes de la supervivencia en compañeros que no se encontraban presentes en la formación, estos saberes fueron avalados y validados por ciertos militantes como cursos de acción propios - pasados o presentes - al momento de las entrevistas individuales.

Finalmente, los saberes de la lucha refieren al acumulado de saber-hacer y saber-deliberar en relación con lo político-organizativo. En el marco de los dispositivos de formación, estos saberes procedían tanto de las prácticas políticas experienciadas por los militantes como de saberes transmitidos por sus familias o por militantes de otras generaciones políticas. Estos saberes competen a las tácticas de resistencia, los modos de enfrentar al adversario, las estrategias de negociación con las autoridades estatales, las experiencias de autodefensa, los análisis de coyuntura, el sentido de oportunidad para la acción y la organización de una asamblea, entre otras. Algunas máximas vinculadas a los saberes de la lucha que se hicieron presentes en las formaciones fueron: todo lo que tenemos lo conseguimos luchando, porque "nadie nos regala nada"; cuantos más compañeros participen de una acción de lucha, más fuerte se vuelve el reclamo; el cambio social es posible si se lucha, porque el capitalismo no es el único sistema social posible; "somos más que ellos"; conocer los derechos es fundamental para salir a luchar; analizar la coyuntura es necesario para elegir los momentos, las herramientas, los aliados y los enemigos de la lucha, a veces para luchar hay que esperar primero; nunca ir

14 Estos saberes de la supervivencia se extrajeron de las entrevistas y los registros de observación de los tres casos de estudio. Se privilegiaron aquellos saberes que aparecieron mencionados reiteradamente. 
solo a negociar con el funcionario público, respetar los acuerdos y poner a consideración de la asamblea los resultados de la negociación. ${ }^{15}$

Al igual que en los saberes cotidianos, las intervenciones de los militantes daban cuenta de una memoria de saberes que, en este caso, se remontaba a experiencias - vividas o transmitidas - como la crisis argentina del año 2001, las organizaciones políticas y guerrillas de las décadas del sesenta y setenta, la resistencia peronista y el peronismo histórico. Los militantes extranjeros también aportaron una memoria de saberes de la lucha desde sus historias locales vinculadas a la resistencia de Túpac Amaru II, la guerra de la Triple Alianza, la guerra del Chaco, el Chile de Allende y la Bolivia de Evo Morales. Esta memoria deviene fundamental para operar en el presente tomando los aspectos más potentes de esas experiencias organizativas pasadas y aprendiendo de las lecciones de las derrotas del campo popular.

Si bien los saberes populares fueron parte de los tres talleres de formación política en estudio, se observaron diferencias respecto a la intensidad de su presencia y su combinación con los conocimientos académico-disciplinares y académico-escolares. Si la singularidad de las formaciones del MPLD y el MDS estuvo dada en la conformación de un dispositivo textual, la EMH de la que participó el MNCI-Buenos Aires muestra un modo de configuración alternativo del dispositivo de formación política que aquí llamaremos "narrativo-experiencial", por su remisión a la oralidad y la experiencia. ${ }^{16}$ Lo anterior se basa en el fuerte descentramiento de la formación respecto a los conocimientos académico-disciplinares para estructurarse en torno a la recuperación, el intercambio y la valoración de los saberes "por experiencia vivida".

Esta configuración apareció reflejada en la decisión metodológica de la coordinación de partir de la experiencia de los militantes sin apostar a traducir esas intervenciones en conceptos o abordajes teóricos. De hecho, se observó la ausencia de materiales escritos (cuadernillos, cartillas, textos y libros) de apoyatura a la formación. La metodología consistió en el tránsito de los destinatarios por tres momentos, con eje en el rescate de la experiencia: el momento personal invitó a los militantes a identificar y situar los acontecimientos que marcaron sus historias personales en el marco más

15 La enumeración de saberes de la lucha fue recuperada de las entrevistas y los registros de observación de los tres casos de estudio.

16 Tal como se tematizó en Palumbo (2017), esta configuración alternativa propia del dispositivo pedagógico del MNCI-Buenos Aires podría vincularse con el carácter rural de corte indígenocampesino del encuadre nacional que asume la formación de las distintas organizaciones que nuclea, a diferencia del MPLD y MDS, que se presentan como movimientos populares netamente urbanos. En este sentido, la inscripción rural o urbana no solo da cuenta de ámbitos de desarrollo disímiles, sino también de sujetos pedagógicos y trayectorias escolares diferentes, marcados por la posibilidad de acceso al sistema educativo. Lo anterior condiciona, a su vez, los tipos de saberes disponibles en los dispositivos de formación y la intensidad de la presencia de cada uno para ser puestos en circulación. 
grande de la historia del país; el momento comunitario apuntó a posicionar el momento personal en el contexto del territorio y sus conflictos, y a rescatar el trabajo de base en los barrios y comunidades; y el tercer momento, llamado organizativo, vinculó a la comunidad con el proceso político de construcción de organización mediante la tematización de la experiencia de militancia, el análisis de la coyuntura y la puesta en común de los principios organizativos y las luchas del movimiento.

Adicionalmente, lo experiencial se plasmó en la búsqueda de generar en los destinatarios una nueva experiencia total - de cuerpo y mente-, que se plasmó en la realización de visitas a comunidades indígeno-campesinas cercanas al lugar donde se desarrolló la formación, la participación diaria en místicas planificadas y espontáneas, y el aspecto convivencial que le imprimía la forma campamento. Por su parte, lo narrativo se basó en la primacía de la oralidad, que tuvo en la centralidad adjudicada a los testimonios su ejemplo más vívido, sea en las dinámicas de trabajo grupal o en instancias deliberadamente creadas donde ciertos militantes compartieron con el plenario sus recorridos biográficos, que anudaban lo personal y lo militante.

\section{Reflexiones finales}

El artículo se propuso analizar la trama epistémica convocada en talleres de formación política impulsados desde movimientos populares, aproximándose a ellos en su condición de dispositivos pedagógico-epistémicos. Para ello, tres experiencias formativas llevadas adelante durante el período 2011-2015 fueron tomadas como referentes empíricos. El trabajo de campo realizado arrojó la existencia de tres tipos de conocimientos y saberes en convivencia en los talleres de formación: conocimientos académico-disciplinares, conocimientos académico-escolares y saberes populares.

La hipótesis interpretativa que sostuvimos a lo largo del texto refiere a que los modos de puesta en juego de conocimientos y saberes disponen y jerarquizan ciertas fuentes epistémicas en los talleres de formación. En este sentido, postulamos dos configuraciones alternativas asumidas por los dispositivos pedagógicos. Por una parte, dispositivos textuales estructurados en torno a conocimientos académico-disciplinares o saberes "librescos". Por otra parte, dispositivos narrativo-experienciales basados en la primacía de los saberes populares o saberes "por experiencia vivida".

La consideración respecto a la configuración adoptada por los dispositivos resulta de importancia particularmente en talleres de formación enmarcados en movimientos populares multisectoriales, donde los militantes involucrados no son igualmente portadores de los distintos tipos de conocimientos y saberes descriptos. El conocimiento académico-disciplinar se inscribía en especial en las trayectorias de los militantes de clase media general - aunque no necesariamente - universitaria y, en algunos casos, en 
militantes de base "compañeros de los barrios" con una extensa militancia política, siendo saberes emergentes para gran parte de los militantes de base "compañeros de los barrios" en formación. Por su parte, los conocimientos académico-escolares aparecían como un piso epistémico común a todos los participantes del dispositivo, más allá de las diferencias - a veces marcadas - en sus trayectorias escolares. Finalmente, los saberes populares se presentaban como los saberes consolidados de los militantes de base de extracción barrial, desde los cuales participaban e intervenían en los talleres. Estas diferenciaciones en los conocimientos y saberes de carácter interno a los movimientos deben situarse, a su vez, en un contexto social que adjudica un estatus superior a los conocimientos académico-disciplinares.

En este sentido, la ya mencionada combinación del aporte de las "ciencias" con la recuperación y el intercambio de saberes populares continúa siendo un desafío para las organizaciones populares. El armado de un dispositivo textual debe lidiar con el riesgo potencial de la reintroducción de concepciones elitistas del saber al estructurarse en torno al conocimiento considerado socialmente válido que se encarna, además, en sujetos con ciertas trayectorias educativas y de clase. A su vez, la configuración de un dispositivo narrativo-experiencial debe lidiar con el riesgo potencial de una concepción localista que desestime aquellos conocimientos académico-disciplinares y académico-escolares que puedan ser de utilidad para la transformación social.

Para un movimiento multisectorial y un militante popular, los distintos conocimientos y saberes revisitados en este artículo son importantes y necesarios no solo para la dinámica de formación, sino también para la construcción política cotidiana. Más allá de la documentación de la diversidad de conocimientos y saberes en los talleres de formación política, se impone la necesidad de indagar en las formas de convivencia dentro de la compleja trama epistémica allí presente. De lo contrario, concepciones elitistas o bien localistas del saber podrían ser reproducidas en estos dispositivos epistémicopedagógicos.

\section{Referencias bibliográficas}

Agamben, G. (2011). ¿Qué es un dispositivo? Revista Sociológica, 26(73), pp. 249-264.

Aguiló, V. y J. Wahren (2014). Los Bachilleratos Populares como campos de experimentación social. Revista Argumentos, 74, pp. 97-114.

Bruno, D. (2016). Redefiniciones político, ideológicas e identitarias de las organizaciones populares autónomas. Estudio retrospectivo del discurso del área nacional de formación del FPDS entre 2001 y 2011 [Tesis de Doctorado en Ciencias Sociales]. Buenos Aires: Universidad de Buenos Aires. 
Burgos, A. (2013). Movimientos sociales, resistencia y educación: la escuela agroecología del Movimiento Campesino de Santiago del Estero/vía Campesina [Tesis de Maestría en Educación, Pedagogías Críticas y Problemáticas Socioeducativas]. Buenos Aires: Universidad de Buenos Aires.

Caldart, R. S. (2004). Pedagogia do Movimento Sem Terra. San Pablo: Expressão Popular.

Ceceña, A. E. (2008). De saberes y emancipaciones. En A. E. Ceceña (coord.) (2008). De los saberes de la emancipación y de la dominación. Buenos Aires: CLACSO, pp. 15-35.

De Sousa Santos, B. (2006). Renovar la teoría crítica y reinventar la emancipación social (encuentros en Buenos Aires). Buenos Aires: CLACSO.

Deleuze, G. (1990). ¿Qué es un dispositivo? En E. Balbier y G. Deleuze (1990). Michel Foucault filósofo. Barcelona: Gedisa, pp. 155-163.

Di Matteo, J. (2016). Prácticas pedagógicas en organizaciones sociales. La perspectiva de los educadores populares en organizaciones campesinas e indígenas [Tesis de doctorado]. Luján: Universidad Nacional de Luján.

Di Matteo, J.; A. De Mingo y D. Vila (2012). La recuperación del saber y la cultura: algunos debates en educación popular a partir de la experiencia del MOCASE-VC. Ponencia presentada en las III Jornadas Internacionales de Problemas Latinoamericanos, Universidad Nacional de Cuyo, Mendoza, Argentina.

Elisalde, R. y M. Ampudia (comps.) (2009) Movimientos sociales y educación. Teoría e historia de la educación popular en Argentina y América Latina. Buenos Aires: Buenos Libros.

Falero, A.; A. Casas; A. Brenes; A. Rieiro y B. Rocco (2011). Movimientos sociales y formación político-técnica: reflexiones a partir de cinco casos en América Latina. En Y. Acosta, A. Falero, A. Rodríguez, I. Sans y G. Sarachu (coords.) (2011). Pensamiento crítico y sujetos colectivos en América Latina: perspectivas interdisciplinarias. Montevideo: Trilce.

Forni, P. (2010). Los estudios de caso: Orígenes, cuestiones de diseño y sus aportes a la teoría social. Miríada, 3(5), pp. 1-26.

Gluz, N. (2013). Las luchas populares por el derecho a la educación: experiencias educativas de movimientos sociales. Buenos Aires: CLACSO. 
Grinberg, S. (2008). Educación y poder en el siglo XXI. Gubernamentalidad y pedagogía en las sociedades de gerenciamiento. Buenos Aires: Miño y Dávila.

Guelman, A. (2015). Pedagogía y politica: la formación para el trabajo en los movimientos sociales. El caso de la Escuela de Agroecología del MOCASEVC (2009-2012) [Tesis de Doctorado de la Universidad de Buenos AiresÁrea Ciencias de la Educación]. Buenos Aires: Universidad de Buenos Aires.

Guelman, A. y M. M. Palumbo (2015). Prácticas pedagógicas descolonizadoras en experiencias productivas autogestionadas de movimientos sociales: el principio formativo del trabajo. Revista Interamericana de Educación de Adultos, 37(2), pp. 47-64.

Korol, C. (2007). La formación política de los movimientos populares latinoamericanos. $O S A L, 22$, pp. 227-240.

Korol, C. (2008). La subversión del sentido común y los saberes de la resistencia. En A. E. Ceceña (coord.) (2008). De los saberes de la emancipación y de la dominación. Buenos Aires: CLACSO, pp. 177-191.

Longa, F. (2012). Identidades colectivas y nuevas demandas sociales: las generaciones políticas y la cuestión de género en el Frente Popular Darío Santillán. Ponencia presentada en las VII Jornadas de Sociología, Universidad Nacional de La Plata, La Plata, Argentina.

Maldovan Bonelli, J.; L. Fernández Mouján y E. Ynoub (2019). Las estrategias organizativas, políticas y discursivas de la CTEP en el período 20112018: cambios y continuidades ante el retorno del proyecto neoliberal en Argentina. Ponencia presentada en las XIII Jornadas de Sociología, Universidad de Buenos Aires, Buenos Aires, Argentina.

Martínez Carazo, P. (2006). El método de estudio de caso. Estrategia metodológica de la investigación científica. Pensamiento y Gestión, 20, pp. 165-193.

Mejía Jiménez, M. R. (2015). Educación popular en el siglo XXI. Bogotá: Desde Abajo.

Michi, N. (2010a). Movimientos campesinos y educación. Estudio sobre el Movimiento de Trabajadores Rurales Sin Tierra de Brasil y el Movimiento Campesino de Santiago del Estero MOCASE-VC. Buenos Aires: El Colectivo. 
Michi, N. (2010b). Lo que enamora, lo que contagia, lo que entusiasma. Un análisis sobre la producción y reproducción cultural en el MOCASE VC. Ponencia presentada en las II Jornadas Internacionales de Problemas Latinoamericanos, Universidad Nacional de Córdoba, Córdoba, Argentina.

Palumbo, M. M. (2017). Dinámicas de construcción de subjetividades políticas y epistémicas en dispositivos pedagógicos de formación política. Un estudio en movimientos populares multisectoriales del Área Metropolitana de Buenos Aires [Tesis de Doctorado de la Universidad de Buenos Aires-Área Ciencias de la Educación]. Buenos Aires: Universidad de Buenos Aires.

Rigal, L. (2015). Educación y nuevos movimientos sociales: construcción de pensamiento crítico y protagonismo popular. En D. Suárez, F. Hillert, H. Ouviña y L. Rigal. Pedagogías críticas en América Latina. Experiencias educativas alternativas de educación popular. Buenos Aires: Noveduc.

Simons, M. y J. Masschelein (2011). Subjetivación gubernamental, política y pedagógica. Foucault con Rancière. En: M. Simons, J. Masschelein y J. Larrosa (eds.) (2011). Jacques Rancière. La educación pública y la domesticación de la democracia. Buenos Aires: Miño y Dávila, pp. 107-146.

Varcellino, S. (2017). Pensar el dispositivo escolar en diálogo con la obra de Boaventura de Sousa Santos. Cronos, 18(1), pp. 8-30.

Vasilachis de Gialdino, I. (coord.) (2007). Estrategias de investigación cualitativa. Buenos Aires: Gedisa.

Virno, P. (2008). Gramática de la multitud. Para un análisis de las formas de vida contemporáneas. Buenos Aires: Colihue.

Yin, R. (1994). Case Study Research. Design and Methods, Applied Social Research Methods. Newbury Park: Sage.

\section{Contribución de autoría}

El trabajo en su totalidad fue realizado por María Mercedes Palumbo. 